\title{
The effect of flow and orography on the spatial distribution of the very short-term predictability of rainfall from composite radar images
}

\author{
L. Foresti ${ }^{1,2, *}$ and A. Seed ${ }^{2}$ \\ ${ }^{1}$ Royal Meteorological Institute of Belgium, Brussels, Belgium \\ ${ }^{2}$ Bureau of Meteorology, Centre for Australian Weather and Climate Research, Melbourne, Australia \\ *now at: Royal Meteorological Institute of Belgium, Brussels, Belgium
}

Correspondence to: L. Foresti (loris.foresti@meteo.be)

Received: 27 June 2014 - Published in Hydrol. Earth Syst. Sci. Discuss.: 10 July 2014

Revised: 21 October 2014 - Accepted: 23 October 2014 - Published: 27 November 2014

\begin{abstract}
The spatial distribution and scale dependence of the very short-term predictability of precipitation by Lagrangian persistence of composite radar images is studied under different flow regimes in connection with the presence of orographic features. Data from the weather radar composite of eastern Victoria, Australia, a $500 \times 500 \mathrm{~km}^{2}$ domain at 10 min temporal and $2 \times 2 \mathrm{~km}^{2}$ spatial resolutions, covering the period from February 2011 to October 2012, were used for the analyses. The scale dependence of the predictability of precipitation is considered by decomposing the radar rainfall field into an eight-level multiplicative cascade using a fast Fourier transform. The rate of temporal development of precipitation in Lagrangian coordinates is estimated at each level of the cascade under different flow regimes, which are stratified by applying a k-means clustering algorithm on the diagnosed velocity fields. The predictability of precipitation is measured by its lifetime, which is derived by integrating the Lagrangian auto-correlation function. The lifetimes were found to depend on the scale of the feature as a power law, which is known as dynamic scaling, and to vary as a function of flow regime. The lifetimes also exhibit significant spatial variability and are approximately a factor of 2 longer on the upwind compared with the downwind slopes of terrain features. The scaling exponent of the spatial power spectrum also shows interesting geographical differences. These findings provide opportunities to perform spatially inhomogeneous stochastic simulations of space-time precipitation to account for the presence of orography, which may be integrated into design storm simulations and stochastic precipitation nowcasting systems.
\end{abstract}

\section{Introduction}

The scale dependence of the predictability of the atmospheric flow was already studied by Lorenz (1969), who found that there is an intrinsic predictability limit associated to each scale of motion. Similar conclusions can also be extended to the predictability of precipitation, in particular if considering rainfall fields as emerging from multiplicative cascade processes (Schertzer and Lovejoy, 1987; Marsan et al., 1996).

The intuition that large-scale precipitation features are more predictable than small-scale features can be easily verified empirically using both Lagrangian persistence of radar precipitation patterns and outputs from numerical weather prediction (NWP) models. Zawadzki et al. (1994) found that the decorrelation time of radar precipitation patterns by Lagrangian persistence is dependent on the degree of spatial smoothing. Grecu and Krajewski (2000) also detected that the predictability depends on precipitation intensity, the most intense rain rates being less predictable. Seed (2003) studied the scale dependence of the predictability of precipitation by Lagrangian persistence using a fast Fourier transform (FFT) to decompose the radar rainfall field into a multiplicative cascade. Turner et al. (2004) employed a waveletbased decomposition to filter out the unpredictable scales of a radar-based extrapolation technique. Wavelet decompositions were also exploited for the scale-dependent verification of NWP precipitation forecasts to account for the loss of predictability at small scales (e.g., Casati et al., 2004; Bousquet et al., 2006). Sinclair and Pegram (2005) applied an Empirical Mode Decomposition to iteratively decompose the 
precipitation field into meaningful physical structures from the high to the low frequencies. Surcel et al. (2014) used a Discrete Cosine Transform to study the filtering properties of ensemble averaging and discovered that the ensemble members are completely decorrelated below a certain cutoff scale.

The multifractal and scale-dependent nature of rainfall not only complicates the study of its predictability and the verification of forecasts, but also demands more sophisticated forecasting and downscaling techniques. The Short-Term Ensemble Prediction System (STEPS; Seed, 2003; Bowler et al., 2006) is a stochastic precipitation nowcasting scheme that exploits the multifractal principle by decomposing the radar rainfall field into an eight-level multiplicative cascade with an FFT. The cascade is advected with optical flow in Lagrangian coordinates and stochastically evolves in time according to a hierarchy of auto-regressive processes of order 1 - AR(1) - or 2 - AR(2). This allows accounting for the empirical observation that the rate of temporal evolution of precipitation features is a power law of the scale of the feature, which is known as dynamic scaling (see, e.g., Venugopal et al., 1999; Mandapaka et al., 2009). STEPS estimates the rate of Lagrangian development of the cascade levels in real time, which allows adapting to the predictability of the observed sequence of radar images. This is necessary since the predictability of precipitation exhibits a strong temporal variability as shown by Seed (2003), Germann et al. (2006), and Seed et al. (2013).

Germann et al. (2006) also analyzed the geographical distribution of the predictability of precipitation over the conterminous United States and found a region of longer lifetimes extending from eastern Nebraska to Lake Michigan through Iowa, Wisconsin, and northern Illinois. Berenguer and Sempere-Torres (2013) performed a similar analysis using the European radar composite and discovered the predictability to be seasonally dependent, with higher values over the central part of the UK, central continental Europe, and the Baltic regions. However, such geographical differences are strongly affected by the inhomogeneous quality of the European radar composite between the different countries, which use different hardware, operating wavelength, scanning strategy, and signal processing (Huuskonen et al., 2014). The spatial heterogeneity of the statistical properties of rainfall also poses issues for its multifractal simulation, which traditionally assumes spatial homogeneity of the stochastic process. One way to avoid constructing complicated, spatially heterogeneous models is to separately add a spatial trend to correct a homogeneous multifractal model. This trend should account for the spatial inhomogeneity of the long-term climatological distribution of precipitation, which is often controlled by the presence of orographic features (see, e.g., Pathirana and Herath, 2002; Badas et al., 2006).

The climatology of precipitation over complex orography is strongly controlled by flow direction and air stability (Panziera and Germann, 2010), which can also be exploited to design analogue-based nowcasting techniques (Foresti et al., 2013). The contribution of orography to the precipitation enhancement also seems to be a scale-dependent process. This can be observed by extracting features from a digital elevation model (DEM) at different spatial scales and looking at the spatial distribution of persistent precipitation cells. It appears that orographic features need a certain characteristic size (scale) in order to control the spatial distribution of precipitation patterns (e.g., Foresti et al., 2012).

The goal of this study is to analyze the spatial distribution of the scale-dependent predictability of precipitation by Lagrangian persistence of composite radar images under different flow regimes in connection with the presence of orographic features. Data from the weather radar composite of eastern Victoria, Australia, a $500 \times 500 \mathrm{~km}^{2}$ domain at 10 min temporal and $2 \times 2 \mathrm{~km}^{2}$ spatial resolutions, covering the period from February 2011 to October 2012, are used for the analyses. A k-means clustering algorithm is employed to classify the velocity fields into six main flow regimes and to stratify the evaluation of statistics.

This research is an extension of the study of Foresti and Seed (2014), who analyzed the geographical distribution of the STEPS nowcasting biases using the same radar data set in order to detect regions of systematic precipitation growth and decay. The typical areas of rainfall growth and decay due to orographic forcing should be observed also in the spatial distribution of the predictability of rainfall. The orographic forcing is expected to control the spatial distribution of the predictability of precipitation at the meso-gamma $(2-20 \mathrm{~km})$ and partly the meso-beta $(20-200 \mathrm{~km})$ scales, which are smaller than the continental scales analyzed in the literature (e.g., Germann et al., 2006; Radhakrishna et al., 2012; Berenguer and Sempere-Torres, 2013).

The dependence of the dynamic scaling relationship on flow regimes is also studied to test whether there are weather regimes that are more predictable than others. On the other hand, the geographical distribution of the spatial power spectrum is analyzed to explore the degree of spatial scaling of precipitation over the forecast domain. The findings of this study should increase our understanding of the predictability of precipitation by Lagrangian persistence of radar images, which is essential to improve its very short-term forecasting, space-time stochastic simulation, and statistical downscaling.

The paper is structured as follows. Section 2 describes the radar rainfall data set. Section 3 details the methodology. Section 4 illustrates the obtained results and interpretations, while Sect. 5 concludes the paper, and discusses potential improvements and future research perspectives.

\section{Radar rainfall data set}

Data from the weather radar composite of Eastern Victoria, Australia were used for the analyses (see Fig. 1 for the 
domain and the radar locations). The composite merges data from four weather radars located at Melbourne (operating at S-band), Yarrawonga (C-band), Gippsland (C-band) and Canberra-Captains Flat (S-band). The period under analysis is from 15 February 2011 to 31 October 2012.

The operational radar data processing chain for quantitative precipitation estimation (QPE) at the Australian Bureau of Meteorology consists of the following steps:

- Ground clutter removal with Doppler filtering at the radar site.

- Additional ground clutter filtering based on a static clutter map and on the gradients of the vertical profile of reflectivity.

- Beam blockage correction using a DEM to correct for the lost power due to the interception of the radar beam with orography.

- Estimation of the vertical profile of reflectivity using data within a range of $50 \mathrm{~km}$ from the radar.

- Interpolation of the volumetric data into constant altitude plan position indicators (CAPPIs). CAPPIs are computed at a height of $1000 \mathrm{~m}$ using the 3dimensional anisotropic Kriging technique of Seed and Pegram (2001).

- Application of a different climatological $Z-R$ relationship for stratiform and convective rain based on the Steiner classification (Chumchean et al., 2008).

- Compositing operation involving a linear combination of the radar measurements in the overlapping regions as a function of distance from the radar.

- Mean field bias correction with respect to rain gauge measurements using a Kalman filtering approach for its temporal update (Chumchean et al., 2006b).

The final product is a $256 \times 256$ grid with a spatial resolution of $2 \mathrm{~km}^{2} \times 2 \mathrm{~km}^{2}$ and a temporal resolution of $10 \mathrm{~min}$ in a Gnomonic projection. More details on the operational QPE chain at the Australian Bureau of Meteorology are given in Chumchean et al. (2006a, b, 2008) and Seed et al. (2007).

These pre-processing steps are not sufficient to completely remove the radar measurement errors, especially over mountainous regions. The two sources of errors that are the most critical for the analysis of the precipitation predictability are the range dependence of estimated rainfall rates and the reduced visibility in the inner Victorian Alps. In addition, the compositing operation generates some discontinuities in the regions of overlapping radar measurements. Rainfall could also be slightly underestimated in a radius of $\sim 20-30 \mathrm{~km}$ around the radar due to the excessive filtering of ground clutter, which also eliminates some precipitation measurements. Precipitation is also underestimated at ranges exceeding $90-100 \mathrm{~km}$ due to the increasing beam width (sampling

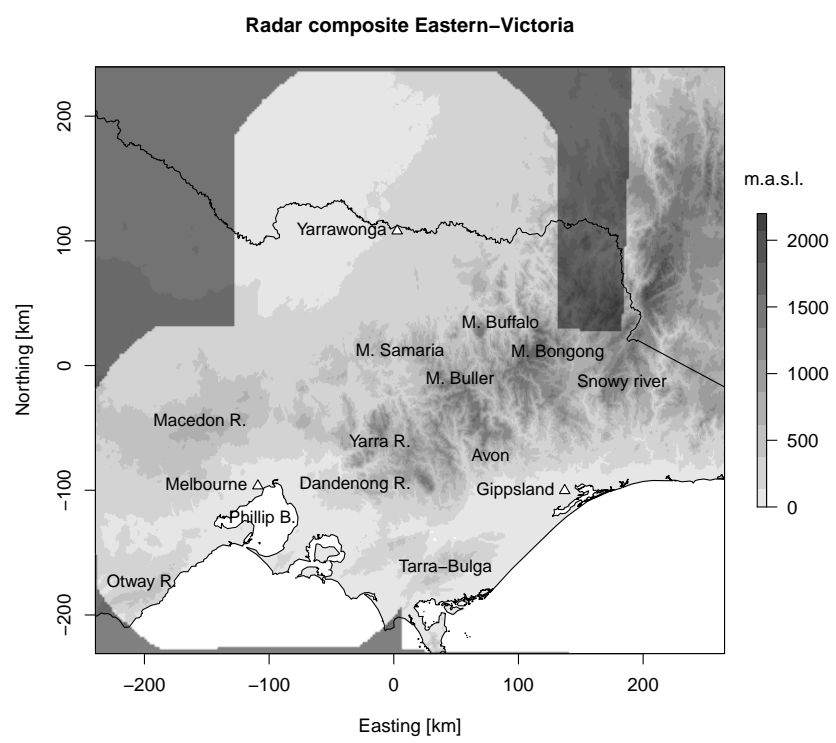

Figure 1. Radar composite of Eastern Victoria, Australia, overlaid on the DEM. Triangles denote the locations of the three radars at Melbourne, Yarrawonga, and Gippsland. In the top-right corner of the domain there is some contribution from the Canberra radar. White tones represent the ocean.

volume), attenuation by rainfall and blockage by orographic features. Hence, precipitation accumulations are strongly underestimated in the inner part of the Victorian Alps where the correction for the vertical profile of reflectivity is evidently not sufficient to extrapolate the higher elevation measurements to the elevation of the CAPPI.

\section{Methodology}

Section 3.1 explains the cascade decomposition framework for the analysis of the scale dependence of the predictability of precipitation. Section 3.2 details the method for estimating the Lagrangian temporal auto-correlation of precipitation, which is needed to evaluate its lifetime (Sect. 3.3). The simultaneous calculation of the Lagrangian auto-correlation at each point of the radar grid using rules for the online computation of the covariance is presented in Sect. 3.4. Section 3.5 presents a simplified approach to estimate the slope of the power spectrum from the variance of the cascade levels under the scaling hypothesis. Finally, Sect. 3.6 provides a brief summary of the k-means stratification of optical flow fields.

\subsection{Cascade decomposition framework}

The radar rainfall field is decomposed using an FFT into a multiplicative cascade of the form (Seed, 2003; Bowler et 
al., 2006):

$\mathrm{dBR}_{i j}=\sum_{k=0}^{K-1} X_{k i j}$ for $i=1, \ldots, L$ and $j=1, \ldots, L$,

where $L=384$ is the size of the squared domain and $K=8$ is the number of cascade levels. A buffer of 64 pixels is added at each side of the original $256 \times 256$ grid in an attempt to reduce the edge effects arising from the FFT transformation, thus giving a larger domain of $384 \times 384$ pixels. The cascade is multiplicative when rewritten in terms of original rain rates $R$ instead of the multiplicative decibel scale $\mathrm{dBR}$. The cascade decomposition is achieved by applying a Gaussian band-pass filter to isolate a given set of spatial scales in the frequency domain (Seed, 2003; see Fig. 2). $X_{k}$ will be referred to as cascade level and is obtained by applying an inverse FFT to the filtered data in order to return the Fourier components back into the spatial domain. Thus, $X_{k}$ represents the variability of the original radar field with spatial frequencies $\left[\mathrm{km}^{-1}\right]$ in the range $q^{k-1} / L<\omega_{k}<q^{k+1} / L$, where $\omega_{k}$ is the central frequency of the Gaussian filter and $q=2.12$ is the branching number (inverse of the scale reduction factor). Each level of the cascade is normalized to zero mean and unit variance for convenience and the normalization is kept constant in space and during the forecast period.

Figure 2 illustrates the Gaussian band-pass filters that are used to isolate the spatial scales composing the set of cascade levels. Given the size of the extended radar domain, an eight-level multiplicative cascade with the following spatial scales is obtained (see Fig. 2): 768-362, 362-171-81, 171-81$38,81-38-18,38-18-8,18-8-4,8-4-2$ and $4-2 \mathrm{~km}$. The noninteger scales resulting from the non-integer branching number of 2.12 were rounded. The scales on which the Gaussian filters are centered are marked in italic. The first and last levels of the cascade will not be considered in the analyses because of not having a regular Gaussian shape. In addition, the largest scale is not able to capture the appropriate scales since the radar composite only covers a certain fraction of the $512 \times 512 \mathrm{~km}^{2}$ domain. This would lead to the underestimation of the precipitation lifetime at that scale (see Sect. 3.3).

\subsection{Lagrangian temporal auto-correlation}

The Lagrangian temporal auto-correlation is a measure for the rate of development of precipitation in storm coordinates and consequently of its predictability (Zawadzki, 1973). An efficient way to follow the rainfall evolution in storm coordinates is to estimate a velocity field using a sequence of radar rainfall fields. STEPS uses an optical flow algorithm (Bowler et al., 2004) for the estimation of the velocity field and a semi-Lagrangian backward-in-time scheme for its advection, which keeps the velocity field fixed and retrieves the rainfall values upstream by following the lines of the velocity field (e.g., Germann and Zawadzki, 2002).

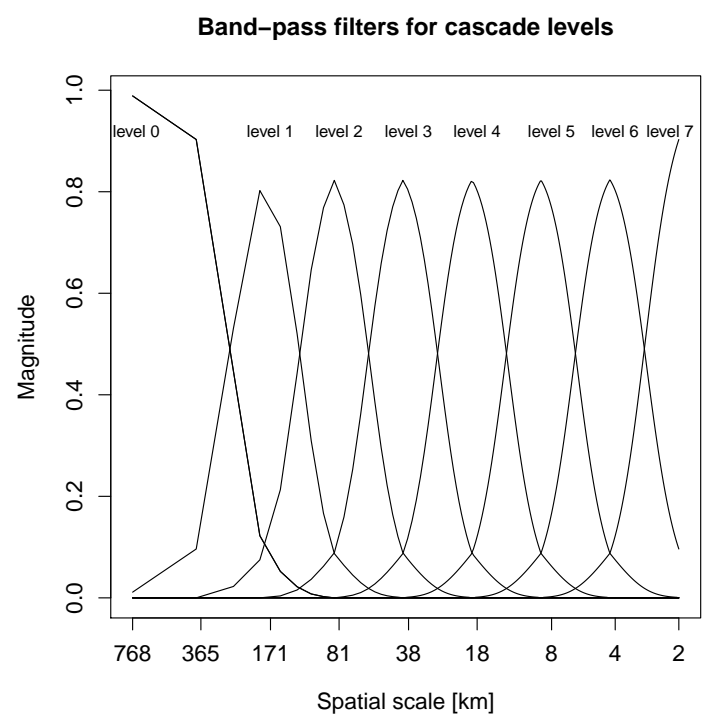

Figure 2. The set of eight Gaussian band-pass filters used to isolate the spatial frequencies composing the cascade levels. The total magnitude for a given spatial frequency is normalized to one.

The Lagrangian lag 1 temporal auto-correlations at each level of the cascade are estimated as follows (Bowler et al., 2006):

1. Estimate the velocity field with optical flow using rainfall fields at time $t-1$ and $t$.

2. Decompose the radar rainfall field at time $t-1$ using FFT into a multiplicative cascade.

3. Decompose the radar rainfall field at time $t$ using FFT into a multiplicative cascade.

4. Advect the cascade from time $t-1$ to time $t$. Note that each level of the cascade is advected with the same velocity field computed on the original rainfall fields.

5. The lag 1 Lagrangian temporal auto-correlation is simply obtained by computing the correlation coefficient between each cascade level $k$ advected from time $t-1$ to $t$ and the corresponding cascade level at time $t$ :

$$
\begin{aligned}
\rho_{1}(k)= & \frac{\frac{1}{L \cdot L} \sum_{i=1}^{L} \sum_{j=1}^{L}\left(X_{k i j}-\bar{X}_{k}\right) \cdot\left(X_{k i j}^{\mathrm{adv}}-\bar{X}_{k}^{\mathrm{adv}}\right)}{\sqrt{\frac{1}{L \cdot L} \sum_{i=1}^{L} \sum_{j=1}^{L}\left(X_{k i j}-\bar{X}_{k}\right)} \sqrt{\frac{1}{L \cdot L} \sum_{i=1}^{L} \sum_{j=1}^{L}\left(X_{k i j}^{\mathrm{adv}}-\bar{X}_{k}^{\mathrm{adv}}\right)}} \\
& \text { for } k=1, \ldots, K-1,
\end{aligned}
$$

where $L=256$ is the size of the radar domain and "adv" refers to the previous value advected forward to the current time. The smaller the correlation coefficients, the higher are the growth and decay of rainfall processes occurring in Lagrangian frame of reference. The lag $2 \mathrm{La}-$ grangian temporal auto-correlation could be estimated 
as well by advecting a cascade at time $t-2$ to time $t$, but is not presented in this paper.

Equation (2) is the ordinary Pearson's correlation coefficient, which involves the subtraction of the field mean. On the other hand, Zawadzki (1973) and Germann and Zawadzki (2002) employed a correlation estimation without subtraction of the mean for estimating the decorrelation time of precipitation fields. The difference between the two approaches is not very important over continental scales, where the forecast and observed fields have similar mean values, but it may become an issue over smaller domains, where the observed mean field precipitation can be significantly different than the forecast one (see, e.g., Foresti et al., 2012). In such a case, Eq. (2) would give lower but more realistic correlation coefficients compared with Germann and Zawadzki (2002).

The Lagrangian auto-correlation estimations are also affected by the presence of different scales of motion. A multiscale optical flow estimation at each level of the cascade may be foreseen but could cause algorithm convergence issues when one is trying to correlate the small-scale features. Also, it is not yet clear how to avoid the appearance of artifacts in the final reconstructed rainfall field when advecting the cascade levels with different velocity fields over several time steps.

Note that the correlation function of Eq. (2) is obtained by integrating over space, i.e., over the total number of pixels $L \cdot L$ within a radar image. This allows the Lagrangian auto-correlation to be estimated in real time and to adapt to the predictability of the sequence of radar images. This approach, however, assumes the predictability to be homogeneous over the forecast domain. Section 3.4 will explain how to obtain estimates of the Lagrangian auto-correlation by performing the summations through time, which is a necessary step for analyzing its spatial distribution.

The hierarchy of Lagrangian temporal auto-correlations defines a hierarchy of auto-regressive processes of order 1 $\mathrm{AR}(1)$. This is exploited by STEPS to stochastically simulate the rainfall growth and decay processes that occur in storm coordinates at different spatial scales to reproduce the dynamic scaling of the field (Seed, 2003; Bowler et al., 2006). The procedure consists of blending the radar cascade with a cascade of spatially and temporally correlated stochastic noise. The spatially correlated noise field is generated using a power law filter while temporal correlations are maintained by a hierarchy of auto-regressive processes. The power law filter ensures that the noise cascade has the same power spectrum of the observed radar rainfall fields. This technique was already employed to generate continuous multifractals (Schertzer and Lovejoy, 1987) and also appeared in the nowcasting system SBMcast (Berenguer et al., 2011), based on the "String of Beads" model of Pegram and Clothier (2001a). The stochastic simulations are stationary and no attempt is made to actually forecast temporal trends in growth and decay of precipitation. Indeed, trying to predict growth and de- cay processes using as predictor the past evolution of radar precipitation does not seem to significantly improve the forecast accuracy, except for the regions characterized by systematic orographic forcing (see a review in Foresti and Seed, 2014). In addition, Radhakrishna et al. (2012) showed that the predictability of growth and decay patterns is 10 times shorter than that of precipitation fields and is limited to spatial scales of the order of $250 \times 250 \mathrm{~km}^{2}$, which would require continental-scale radar images to be studied properly. The stochastic simulations are not presented in this paper but only explained for completeness since they are based on the Lagrangian auto-correlation coefficients.

\subsection{Estimation of the precipitation lifetime}

By knowing the lag 1 auto-correlation coefficient, the AR(1) auto-correlation function $(\mathrm{ACF})$ can be recursively derived as follows:

$\rho(t)=\rho_{1}^{t}$ for $t=1, \ldots, T$,

where $\rho_{1}$ is the lag 1 Lagrangian auto-correlation coefficient computed with Eq. (2). Note that this simplification indirectly assumes that the diagnosed velocity field does not change during the forecast period. In fact, it extrapolates the whole ACF knowing only the lag 1 auto-correlation. This assumption is reasonable up to $2-3 \mathrm{~h}$ (Germann et al., 2005) and 3-4 h lead times (Bowler et al., 2006), since using the correct velocity does not reduce the forecast errors much. A complete study of the Lagrangian predictability of precipitation including the non-stationarity of the velocity field, would involve the direct calculation of the correlation coefficients at each forecast lead time by comparing the forecasts to the observations (see Germann and Zawadzki, 2002). The basic principle of STEPS is to actually estimate the Lagrangian ACF in real time and allow it to adapt to the predictability of the situation. It would be computationally intensive to estimate the complete ACF using a few hours of radar fields before the analysis time. Eventually, the predictability of the field would be representative of the previous hours and not of the last two or three rainfall fields. The adaptability of the system is particularly important, for example, when the field is rapidly evolving from a convective to a stratiform situation or in the early stages of a new rainfall event.

Finally, the lifetime of precipitation (decorrelation time) can be evaluated by integrating the ACF over time (Zawadzki, 1973; Germann and Zawadzki, 2002):

$$
L T=\int_{0}^{\infty} \rho(t) \mathrm{d} t .
$$

For an exponentially decaying ACF the lifetime is defined as the time at which the ACF falls below the value $1 / e=0.37$ (Zawadzki, 1973). Note that with an exponentially decaying 
function, the integral of Eq. (4) can be analytically derived and is equal to $-\frac{1}{\ln \left(\rho_{0}\right)}$.

In order to generalize the methodology to different ACFs and for the comparison of observed and forecast field at all lead times, Eq. (4) was numerically integrated using the extended Simpson's rule (Press et al., 2007).

\subsection{Online collection of rainfall statistics}

Instead of analyzing the temporal distribution of the Lagrangian auto-correlation by integrating the data over space, we want to analyze its spatial distribution by integrating over time. More precisely, the summations of Eq. (2) need to be done over the number of radar images in the archive, not the number of pixels within a radar image. A joint evaluation of the summations at each pixel in a radar field is intractable as it would require loading the whole archive of rainfall fields into the computer memory to compute the correlations in a single pass. An efficient way to overcome this issue is to exploit rules for the online computation of the mean, the variance and the covariance (Knuth, 1998). The online estimation of the mean is obtained as follows:

$\bar{x}_{t+1}=\bar{x}_{t}+\delta / N$,

where $t$ is the iteration, $\bar{x}_{t+1}$ is the new mean, $\delta=x_{t+1}-\bar{x}_{t}$ is the residual contribution of the new sample $x_{t+1}$ to the old mean $\bar{x}_{t}$ and $N$ is the number of samples.

The online estimation of the variance is obtained similarly as follows:

$q_{t+1}=q_{t}+\delta\left(x_{t+1}-\bar{x}_{t+1}\right)$,

where $q$ is the squared sum of the differences of $x$ from its mean and $\delta=x_{t+1}-\bar{x}_{t}$. The variance is obtained offline by dividing $q$ by the number of samples $N$.

The online computation of the AR(1) Lagrangian temporal auto-correlation is evaluated by keeping track of the sum of squared residuals:

$s_{t+1}=s_{t}+\left(x_{t+1}^{\mathrm{adv}}-\bar{x}_{t}^{\mathrm{adv}}\right) \cdot\left(x_{t+1}-\bar{x}_{t+1}\right)$.

The Lagrangian auto-correlation is obtained offline as:

$\rho\left(x, x^{\mathrm{adv}}\right)=\frac{s}{N \sqrt{\operatorname{Var}(x) \cdot \operatorname{Var}\left(x^{\mathrm{adv}}\right)}}$.

The online computation of statistics using these rules gradually converges towards a stable value as the time progresses. In order to admit temporal fluctuations of the statistics and local smoothing, one can introduce a weight in the recursive equations similarly to the technique of recursive least squares computation.

The technical implementation of the online update of the field statistics is performed by keeping binary files containing the arrays of interim statistics. For each new radar field, the old file is read, updated and rewritten with the new statistics. The statistics are only updated when the rainfall fraction exceeds $5 \%$ over the radar composite and when the four radars are jointly operating. With this criterion we obtained 9578 valid rainfall fields, which roughly correspond to $1600 \mathrm{~h}$ of precipitation over the period spanning from February 2011 to October 2012.

\subsection{Offline spectral slope estimation}

A precipitation field that is scale-invariant (also referred to as scaling) typically exhibits a power spectrum of the form:

$P(f) \propto f^{-\beta}$,

where $f$ is the spatial frequency $\left(\mathrm{km}^{-1}\right)$ and $\beta$ is the scaling exponent (the slope of the power spectrum). The power law behavior of rainfall fields usually appears as a straight line on a graph of the logarithm of the power against the logarithm of the spatial frequency. The slope of the line measures the degree of scaling of the field and is equal to 0 for an unstructured white noise field. The scaling exponent of a 2dimensional rainfall field is often greater than 2 , which complicates its multifractal simulation (see, e.g., Schertzer and Lovejoy, 1987). One possibility to simulate stochastic rainfall fields to obtain $\beta>2$ is to apply a power law filter to a field of white noise as briefly mentioned in Sect. 3.2.

Radar rainfall fields often deviate from the theoretical framework of perfect scale-invariance and typically show a scaling break at frequencies of $15-20 \mathrm{~km}^{-1}$ (see, e.g., Gires et al., 2011; Seed et al., 2013). On the other hand, precipitation fields computed by NWP models have a break around 40-50 km (e.g., Gires et al., 2011). The scaling break is observed as an increase in the spectral slope at the smaller convective scales. This seems to have a physical origin and could be attributed to different scaling regimes of the large-scale stratiform rainfall and the smaller convective scales. However, recent analyses explain this phase transition with the presence of zeros in the field, which also affects the estimation of universal multifractal parameters (Gires et al., 2012). The presence of a scaling break requires using two spectral slopes $\beta_{1}$ and $\beta_{2}$ for the study and parameterization of the power spectrum. $\beta_{1}$ accounts for wavelengths that are larger than $15-40 \mathrm{~km}$ and $\beta_{2}$ for wavelengths that are lower than $15-40 \mathrm{~km}$.

In this research we analyze the spatial distribution of the spectral slopes $\beta_{1}$ and $\beta_{2}$. A complete analysis would require visiting each radar pixel and performing a local spatial FFT decomposition in its neighborhood, which is very computationally demanding if one wants to repeat the analysis over a long period of time. Instead, the two spectral slopes are derived offline from the spatial distribution of the variance at each level of the cascade. This can be achieved by assuming scaling of the variance of the cascade levels (see Menabde et al., 1997). It consists of evaluating the average slope increments between successive levels of the cascade level standard 
Table 1. Characteristics of the six flow cluster centers that are used to stratify the statistics. Wm and Ws refer to moderate and strong westerly flows respectively. The detailed average velocity maps can be found in Foresti and Seed (2014).

\begin{tabular}{lcccccc}
\hline Cluster label & 0-SE & 1-Wm & 2-N & 3-SW & 4-NW & 5-Ws \\
\hline Average flow direction & Southeast & West & North & Southwest & Northwest & West \\
Average flow magnitude $\left(\mathrm{km} \mathrm{h}^{-1}\right)$ & 8.2 & 17.2 & 21.3 & 21.9 & 40.0 & 37.5 \\
\hline
\end{tabular}

deviations:

$$
\begin{gathered}
H=-\frac{1}{K-1} \sum_{k=1}^{K-1} \frac{\log _{10}\left(\frac{\operatorname{sd}\left(X_{k i j}\right)}{\operatorname{sd}\left(X_{(k+1) i j}\right)}\right)}{\log _{10}(q)} \\
\quad \text { and } \beta=2 H+E,
\end{gathered}
$$

where $\operatorname{sd}\left(X_{k i j}\right)$ is the standard deviation of cascade level $k$ at pixel $i j$ and $E=2$ is the dimension of the space. $\beta_{1}$ is estimated using levels 1 to 3 (scales of 171,81 and $38 \mathrm{~km}, K=3$ in Eq. 10) while $\beta_{2}$ using levels 3 to 6 (scales of 38, 18, 8 and $4 \mathrm{~km} ; K=4$ in Eq. 10). A scaling break of $40 \mathrm{~km}$ instead of $20 \mathrm{~km}$ was chosen to obtain smoother fields of the spectral exponent $\beta_{2}$, which is consequently slightly underestimated.

Note that this approach is different than estimating power spectra on rainfall time series and analyzing the spatial distribution of the spectral exponents. The approach proposed in this paper should give insights into the spatial heterogeneity of the degree of spatial scaling of rainfall fields.

\subsection{K-means stratification of optical flow fields}

To analyze the dependence of rainfall statistics on flow regimes, the optical flow fields were stratified using the kmeans clustering algorithm. The details on the preparation of the archive of optical flow fields and the clustering algorithm can be found in Foresti and Seed (2014).

Table 1 summarizes the statistics of the six cluster centers obtained after running the k-means algorithm on the archive of flow fields. The cluster centers mainly differ in terms of flow direction and magnitude, while the spatial variability of the velocity vectors within a field is only marginal (see Foresti and Seed, 2014). The number of clusters was empirically chosen to represent a sufficient number of flow regimes and to have enough samples per cluster to compute significant verification statistics. The cluster 0 is characterized by weak southeasterly winds, the cluster 1 by moderate westerly winds, the cluster 2 by moderate northerlies, the cluster 3 by moderate southwesterly winds, the cluster 4 by strong northwesterly winds, and the cluster 5 by strong westerlies. It is understood that winds refer to the apparent motion of radar images derived with optical flow and not to real wind fields.

The online update of the statistics (Sect. 3.4) is performed by keeping a set of six binary files containing the interim fields of the rainfall mean, variance, Lagrangian autocorrelations and number of samples. The files are read, up- dated and rewritten according to the cluster membership of a given field.

\section{Results}

Section 4.1 illustrates the scale-dependent geographical distribution of the precipitation lifetime without stratification into flow regimes. On the other hand, Sect. 4.2 shows the flow dependence of the dynamic and spatial scaling relationships by averaging the results over space. Finally, Sect. 4.3 analyzes the spatial distribution of the precipitation lifetime under different flow regimes to understand the effect of orographic forcing.

\subsection{Geographical distribution of precipitation predictability and spatial scaling}

Figure 3 illustrates the spatial distribution of the precipitation lifetime for the cascade levels 2, 3, 4, and 5 without stratification into flow regimes. Refer to Fig. 1 for geographical details. The level 0 is not presented since the FFT filter does not have a Gaussian shape (see Fig. 2). On the other hand, the level 1 is too influenced by the edge effects that propagate from the borders of the radar composite towards the interior regions. The levels 6 and 7 are too noisy and exhibit lifetimes that are below the temporal resolution of the radar composite (10 min).

The cascade level 2 (171-81-38 km, Fig. 3a) has lifetimes comprised between 5 and $10 \mathrm{~h}$ but still highlights the presence of some edge effects. The long lifetimes obtained may still be a consequence of assuming the diagnosed velocity field to be temporally stationary. An important part of the spatial variability at this scale is affected by the shape of the radar composite, and long lifetimes tend to be located in its central parts. The other cascade levels (Fig. 3b-d) are less affected by the edge effects, which remain limited to a small region close to the borders of the radar composite. All of them have the longest lifetimes over the flat regions surrounding the Yarrawonga radar. In this region the lifetimes are up to $3.5 \mathrm{~h}$, and 70 and $30 \mathrm{~min}$ for the $81-38-18$, 38-18-8 and 18-8-4 km scales, respectively. These long lifetimes can be explained by a higher Lagrangian predictability over flat continental areas (see for instance Germann et al., 2006). The lifetimes around the Macedon ranges are lower on their southeast flanks in the direction of the Melbourne radar compared with their northwest flanks. Despite being 


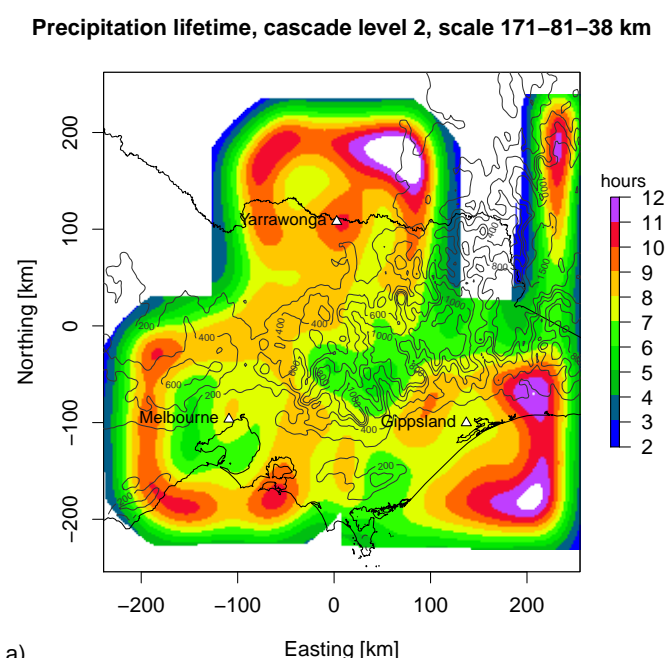

a)

Precipitation lifetime, cascade level 4, scale 38-18-8 km

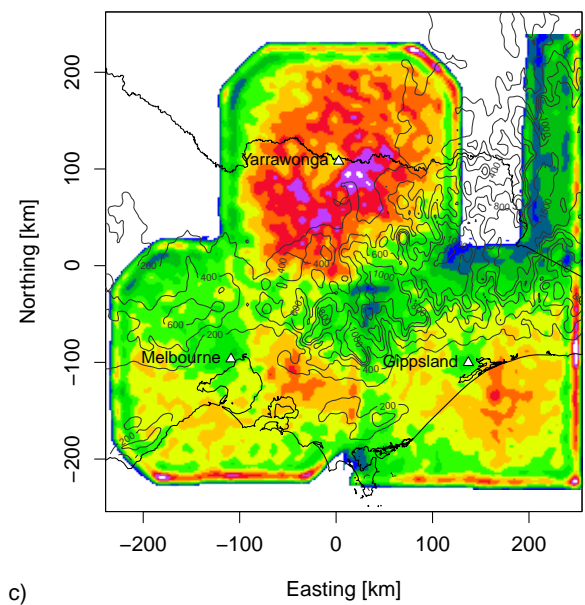

Precipitation lifetime, cascade level 3, scale 81-38-18 km

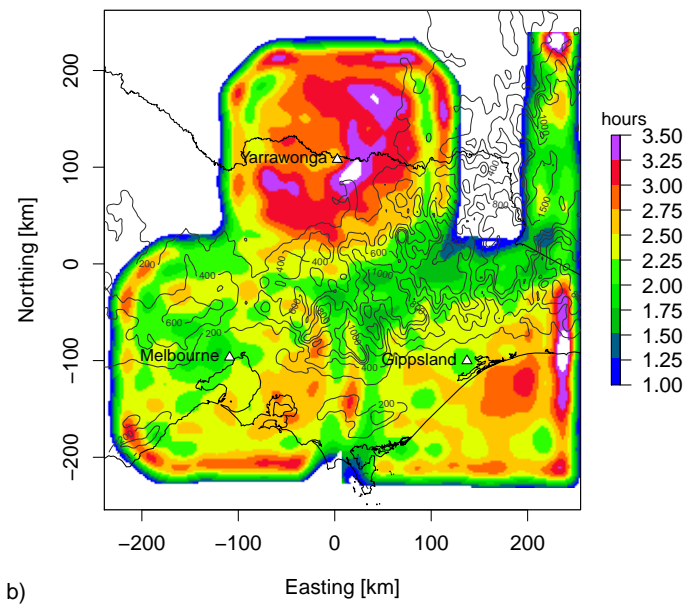

Precipitation lifetime, cascade level 5, scale 18-8-4 km

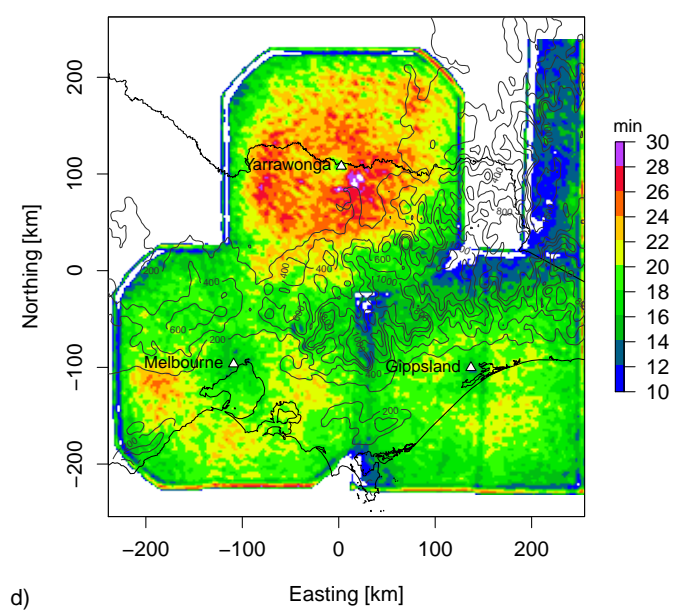

Figure 3. Spatial distribution of the precipitation lifetimes for the four middle cascade levels. (a) 171-81-38 km, (b) $81-38-18 \mathrm{~km}$, (c) $38-18-8$ and (d) $18-8-4 \mathrm{~km}$. White tones are used for regions outside the radar domain or presenting values that exceed the range of the color scale.

less pronounced, this pattern was already observed by Foresti and Seed (2014) and is a consequence of the prevailing westerly flows, which cause systematic rainfall decay on the leeward side of the Macedon ranges and orographic enhancement on their windward side. This effect is also the origin of the long lifetimes observed on the Dandenong ranges as they are located upwind relative to the prevailing westerlies. The lifetimes surrounding the Gippsland radar tend to be longer over the ocean, which is particularly visible in Fig. $3 \mathrm{~b}$ and c. Finally, the shorter lifetimes on the inner parts of the Victorian Alps are probably due to the reduced accuracy of the radar measurements (see Sect. 2). In particular, the blockage of radar beams, the rainfall attenuation and overshooting reduce the accuracy of the optical flow estimations, which consequently affects the lifetimes derived from the Lagrangian auto-correlation. In addition, it seems that there is a proportional effect between the precipitation lifetime and the cli- matological precipitation amount: the lifetimes are generally lower in the places where the radar measures less precipitation (see e.g., Berenguer and Sempere-Torres, 2013).

Figure 4 illustrates the spatial distribution of the spectral slopes $\beta_{1}$ and $\beta_{2}$ derived from the standard deviation of the cascade levels (see Sect. 3.5). $\beta_{1}$ represents the degree of scaling above the $40 \mathrm{~km}$ scale; $\beta_{2}$ represents that below it and they therefore account respectively for the large-scale precipitation structures and convective features. Except for the regions close to the radar domain edges, $\beta_{1}$ is generally larger than 2 with the highest values in the range 2.2-2.4 and centered on the three radars. These are the regions where the spatial scaling of rainfall can be measured more efficiently and is the highest. At first sight, these inhomogeneities can only be explained by the shape of the radar composite and not by the presence of different atmospheric processes. However, the spectral slopes are higher on the southern slope of 

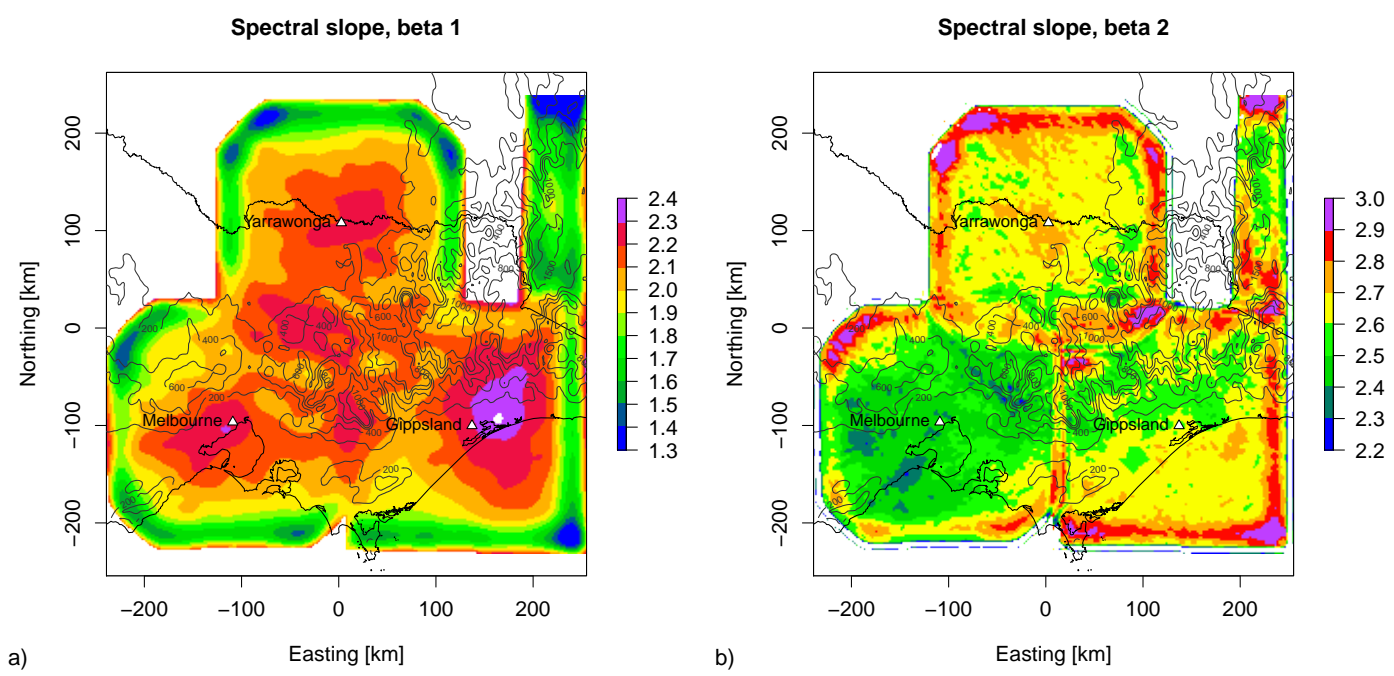

Figure 4. Spatial distribution of the spectral slopes (a) $\beta_{1}$ and (b) $\beta_{2}$ derived by assuming the scaling of the standard deviation of the cascade levels.

the Alps located northeast of the Gippsland radar. This depicts a region characterized by rainfall fields that are highly organized in space with convection embedded into stratiform rainfall, which is typical of orographic rainfall (see Fig. 6a). It would be interesting to perform a similar analysis using outputs from NWP models to eliminate the heterogeneities introduced by the inhomogeneous quality of radar measurements. As expected, the spectral slopes at the small scales $\left(\beta_{2}\right.$, Fig. $\left.4 \mathrm{~b}\right)$ are systematically higher than the ones at the large scales $\left(\beta_{1}\right.$, Fig. $\left.4 \mathrm{a}\right)$, with values in the range 2.3-2.8. However, the spectral slope $\beta_{2}$ is lower in the surroundings of the Melbourne radar (S-band) compared with the other two (C-band). Both the $\mathrm{C}$ - and S-band radars have a $1^{\circ}$ azimuth and $250 \mathrm{~m}$ range resolution (see for instance, Rennie, 2012). Notwithstanding the same resolution, the rainfall field exhibits more power in the last cascade level in the surroundings of the Melbourne radar, which can explain the lower spectral exponent $\beta_{2}$ (Fig. 4b). The patterns observed in Fig. $4 \mathrm{~b}$ are hard to explain in terms of different precipitation regimes and seem to be more associated to the type of radar or data processing chain. Despite these differences, the spectral exponents $\beta_{2}$ tend to be lower upwind than upstream of the mountain ranges, in particular over the Yarra and Dandenong ranges, the southern slopes of the Alps between Avon and the Snowy River, and on the northern slopes of the Alps located southeast of the Yarrawonga radar. This depicts that strong convection is more likely to occur over flat regions than over complex orography, where it is less intense and often embedded into stratiform rainfall.

\subsection{Flow dependence of the dynamic and spatial scaling relationships}

Table 2 and Fig. 5 illustrate the dynamic scaling relationship between the spatial scale and the precipitation lifetime for each flow regime. As already explained in Sect. 3.1, only the cascade levels 1 to 6 are shown. The values are obtained by spatial averaging of the lifetimes within the radar composite. Figure 5 demonstrates the presence of dynamic scaling, which is observed as a clear power law relationship between the spatial scale of precipitation features and its estimated lifetime (Venugopal at al., 1999; Seed, 2003). It is worth mentioning that Venugopal at al. (1999) employed another statistical quantity to account for the temporal evolution of rainfall and the obtained dynamic scaling exponents cannot be directly compared. The figure also shows significant variability of the lifetimes as a function of flow regime. The clusters NW and Ws are characterized by the shortest lifetimes, and the cluster SE by the longest. These differences are in part due to the type of rainfall, which is more convective under northerly than southerly flows. In fact, the organized convective activity mostly occurs when the warm continental northwesterly flows meet the colder maritime air. On the other hand, it is not clear whether the faster translational speed of convective rain relative to stratiform rain affects the estimation of the predictability by Lagrangian persistence. These lifetime estimations are a bit higher than the original ones of Seed (2003), who used a single motion vector to advect the radar rainfall field. Similar issues were encountered by Pegram and Clothier (2001b) because of using a single displacement vector and as a consequence of the high level of noise at the pixel scale. This demonstrates the added value of the optical flow algorithm of Bowler et al. (2004), which better defines the differential motion within a rainfall field, as well as the analysis of the Lagrangian predictability at larger scales using the Fourier-based scale decomposition. The estimations also compare well with the results of Germann et al. (2006), who reported lifetimes of $0.1-0.2 \mathrm{~h}$ on the $4-8 \mathrm{~km}$ scale using a wavelet decomposition of the rainfall 
Table 2. Precipitation lifetimes for each spatial scale and flow regime averaged over the radar composite. Levels $0-3$ are expressed in hours and 4-7 in minutes. The power law extrapolation of lifetimes for smaller spatial scales is given in seconds. Ext.: estimation of the lifetimes at smaller spatial scales by extrapolating the power law. The extrapolation uses the original non-integer scales for increased precision. The scales on which the Gaussian filters are centered are marked in italic.

\begin{tabular}{lllllllll}
\hline Level & $\begin{array}{l}\text { Spatial scales } \\
{[\mathrm{km}]}\end{array}$ & $\begin{array}{l}\text { Cluster 0 } \\
\mathrm{SE}\end{array}$ & $\begin{array}{l}\text { Cluster } 1 \\
\mathrm{Ww}\end{array}$ & $\begin{array}{l}\text { Cluster 2 } \\
\mathrm{N}\end{array}$ & $\begin{array}{l}\text { Cluster 3 } \\
\text { SW }\end{array}$ & $\begin{array}{l}\text { Cluster 4 } \\
\text { NW }\end{array}$ & $\begin{array}{l}\text { Cluster 5 } \\
\text { Ws }\end{array}$ & $\begin{array}{l}\text { Weighted } \\
\text { average }\end{array}$ \\
\hline 0 & $768-362$ & 27.7 & 25.9 & 28.5 & 25.4 & 19.3 & 20.2 & $24.1 \mathrm{~h}$ \\
1 & $362-171-81$ & 24.9 & 21.4 & 18.6 & 22.5 & 14.6 & 16.0 & $19.2 \mathrm{~h}$ \\
2 & $171-81-38$ & 13.7 & 8.9 & 7.9 & 9.6 & 5.6 & 6.3 & $8.3 \mathrm{~h}$ \\
3 & $81-38-18$ & 4.6 & 2.8 & 2.5 & 3.1 & 1.8 & 2.0 & $2.7 \mathrm{~h}$ \\
4 & $38-18-8$ & 79.6 & 53.9 & 48.9 & 56.3 & 34.4 & 38.7 & $49.8 \mathrm{~min}$ \\
5 & $18-8-4$ & 26.4 & 21.0 & 19.2 & 20.5 & 14.0 & 15.7 & $19.0 \mathrm{~min}$ \\
6 & $8-4-2$ & 10.5 & 9.2 & 8.5 & 8.7 & 6.4 & 7.31 & $8.3 \mathrm{~min}$ \\
7 & $4-2$ & 5.9 & 5.0 & 5.2 & 5.4 & 4.7 & 5.0 & $5.1 \mathrm{~min}$ \\
\hline ext. 8 & $1.89-0.89-0.42$ & 78 & 65 & 62 & 58 & 43 & 49 & $58.0 \mathrm{~s}$ \\
ext. 9 & $0.89-0.42-0.20$ & 28 & 23 & 23 & 16 & 16 & 18 & $20.5 \mathrm{~s}$ \\
ext. 10 & $0.42-0.20-0.09$ & 10 & 8 & 8 & 5 & 6 & 7 & $7.4 \mathrm{~s}$ \\
\hline
\end{tabular}

field over the continental United States. In Fig. 5 the $4-8 \mathrm{~km}$ scales roughly correspond to the $8-4-2 \mathrm{~km}$ and $18-8-4$ scales, which exhibit lifetimes of $0.1-0.4 \mathrm{~h}$.

To obtain an order of magnitude for the predictability at smaller spatial scales, power law relationships were fitted using the method of least squares per each flow cluster. The extrapolation of the fitted power laws towards smaller spatial scales could give an idea of the minimum temporal resolution that is required to reliably measure the Lagrangian auto-correlation of precipitation, which is very important for stochastic precipitation nowcasting at urban scales (e.g., Goormans and Willems, 2013; Ruzanski and Chandrasekar, 2012). The bottom of Table 2 shows the results of such extrapolations for scales of 1.89-0.89-0.42, $0.89-0.42-0.20$ and $0.42-0.20-0.09 \mathrm{~km}$. Because of working on a logarithmic scale such estimations are quite uncertain and to a certain degree pessimistic, in particular because the dynamic scaling relationship does not perfectly follow a power law. The imperfect dynamic scaling could also be due to using the lifetime instead of the temporal rainfall changes as a measure for the rainfall evolution (see Venugopal et al., 1999). It must also be considered that the optical flow is representative of the scales measured by the C- and S-band radars and cannot capture the motion at smaller scales. From this simple extrapolation, the kilometric scale (1.89-0.89$0.42 \mathrm{~km}$ ) only displays a predictability of $40-80 \mathrm{~s}$. It would be interesting to study whether the temporal resolution of Xband radars is sufficient to reliably measure the Lagrangian auto-correlation of the very small scale precipitation features. Using such high resolution data will also pose the computational challenge of generating the nowcasts before the predictability limits have been exceeded to avoid the forecasts becoming obsolete. Ruzanski and Chandrasekar (2012) re- ported a predictability of $20 \mathrm{~min}$ using data from a network of $\mathrm{X}$-band radars and the CASA nowcasting system. The scale dependence was analyzed by upscaling the forecasts and the values are not directly comparable to the ones obtained by scale separation within STEPS. At these temporal scales, the quality of the nowcasts is still strongly affected by the accuracy of the input radar observations. Therefore, it becomes necessary to complement nowcasting systems with heuristic models of the radar measurement uncertainty, for example to account for stochastic sampling errors (Jordan et al., 2003).

Table 3 illustrates the spectral slopes $\beta_{1}$ and $\beta_{2}$ of the spatial power spectrum stratified by flow regime. $\beta_{1}$ typically oscillates around the dimension of the field with the smallest values occurring under the flows SE-SW (1.88-1.91) and the largest under the flows $\mathrm{Wm}, \mathrm{N}$, and NW (2.01-2.03). The values are slightly smaller than the ones found in the literature (e.g., Seed et al., 2013), which is explained again by the presence of edge effects that locally reduce the spectral exponents (see Fig. 4a). This may have consequences on the power law filtering performed by STEPS to generate the noise cascade needed to update the hierarchy of autoregressive processes. In fact, the filtering uses the spatial power spectrum of rainfall as target distribution, which does not account for the spatial heterogeneities within the forecast domain.

The values of $\beta_{2}$ are significantly higher and oscillate between 2.45 and 2.8. The clusters NW and Ws have the highest $\beta_{2}$ (2.68-2.79), which can be attributed to a higher convective activity occurring under these flow conditions. The cluster NW also has a high $\beta_{1}$ and is the one having the most organized rainfall structures from the large down to the small convective scales. 
Table 3. Average spatial spectral exponents stratified by flow regime. The standard deviation over space is given in brackets.

\begin{tabular}{lcccccc}
\hline Cluster label & 0-SE & 1-Wm & 2-N & 3-SW & 4-NW & 5-Ws \\
\hline$\beta_{1}$ & $1.88(0.29)$ & $2.02(0.24)$ & $2.03(0.25)$ & $1.91(0.22)$ & $2.03(0.24)$ & $1.96(0.23)$ \\
$\beta_{2}$ & $2.46(0.20)$ & $2.55(0.15)$ & $2.61(0.18)$ & $2.46(0.19)$ & $2.79(0.16)$ & $2.68(0.18)$ \\
\hline
\end{tabular}

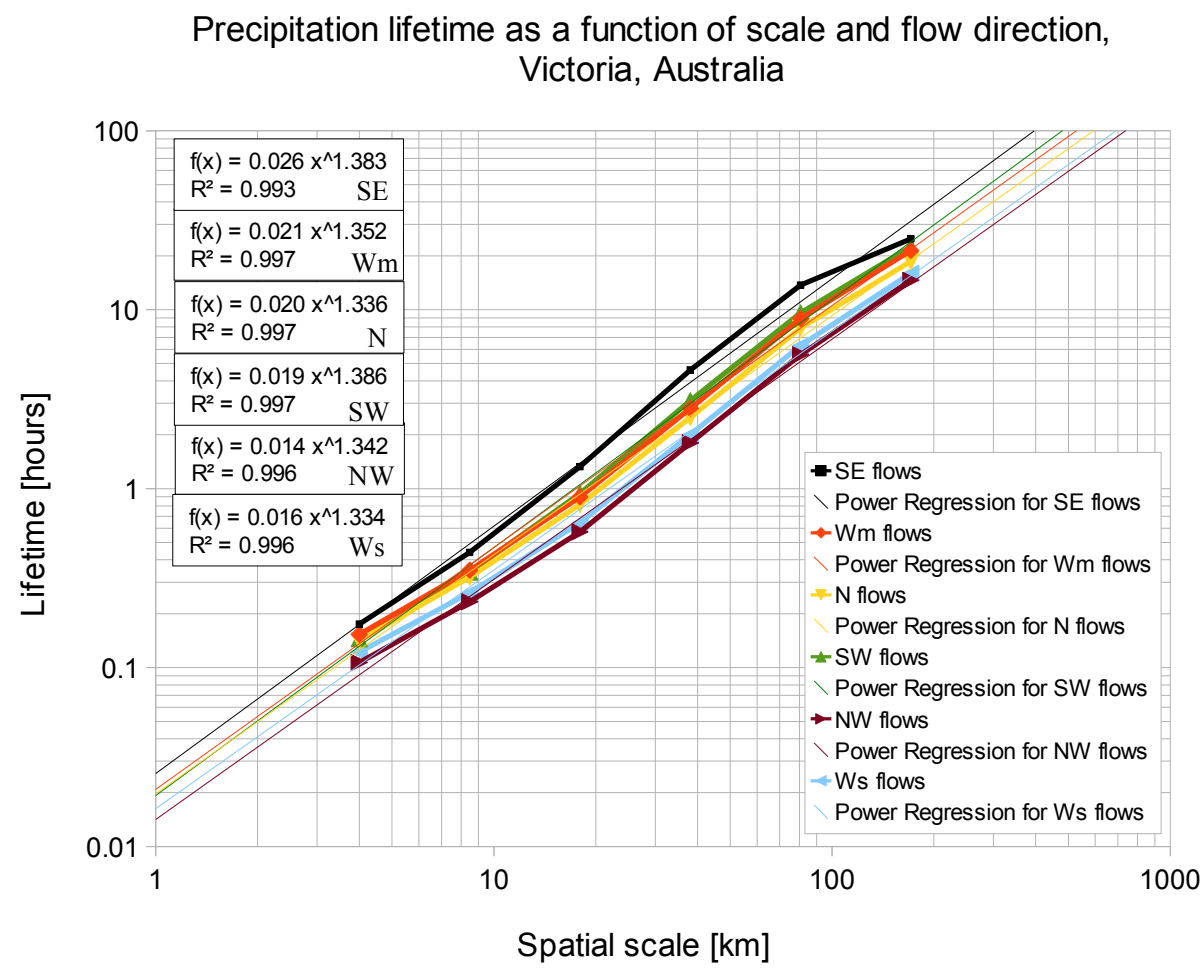

Figure 5. Dynamic scaling relationship between the spatial scale and precipitation lifetime stratified by flow regime. The equations of the power law fits are shown in the upper left corner.

\subsection{Effect of orography on the predictability of precipitation}

According to the results of Berenguer and SempereTorres (2013), the regions of long predictability seem to be correlated with the regions with the highest rainfall accumulations. In fact, the regions that are often affected by organized large-scale precipitation systems are more likely to exhibit higher predictability than the ones with infrequent isolated convection. It is therefore important to analyze the climatology of precipitation to study the spatial distribution of its predictability.

Figure 6 shows the conditional mean 10 min rainfall accumulations stratified by flow regime. It clearly illustrates the flow dependence of the spatial distribution of precipitation, which is mostly located on the windward side of mountain ranges. Most of the precipitation occurring under southeasterly flows is located along the upwind side of the Victorian Alps in a region going from Avon to the Snowy River (Fig. 6a). The spatial distribution of rainfall under moderate westerly flows presents maxima on the Dandenong and Macedon ranges, but also on the northern side of the Alps around Mount Buffalo (Fig. 6b). The enhancement on the Northwest flank of the Alps is much more pronounced with northerly and northwesterly flows, which approach the mountain range more perpendicularly (Fig. $6 \mathrm{c}$ and e, respectively). Southwesterly flows lead to high accumulations on the Yarra and Dandenong ranges as well as the southern side of the Alps around the Gippsland radar (Fig. 6d). It is interesting to note that northwesterly flows also give high accumulations on the leeside of the Alps (Fig. 6e), which could be caused by the lower air stability of these conditions (refer to Foresti and Seed, 2014, for a more detailed interpretation). Finally, strong westerly flows lead again to high accumulations on the Dandenong and Macedon ranges, but also on the West of the Gippsland radar (Fig. 6f). A clear rainfall shadow effect on the leeside of the Macedon ranges is noticed for the clusters Wm, NW, and Ws.

Figure 7 shows the spatial distribution of the precipitation lifetime at the convective scale $(38-18-8 \mathrm{~km})$ stratified 

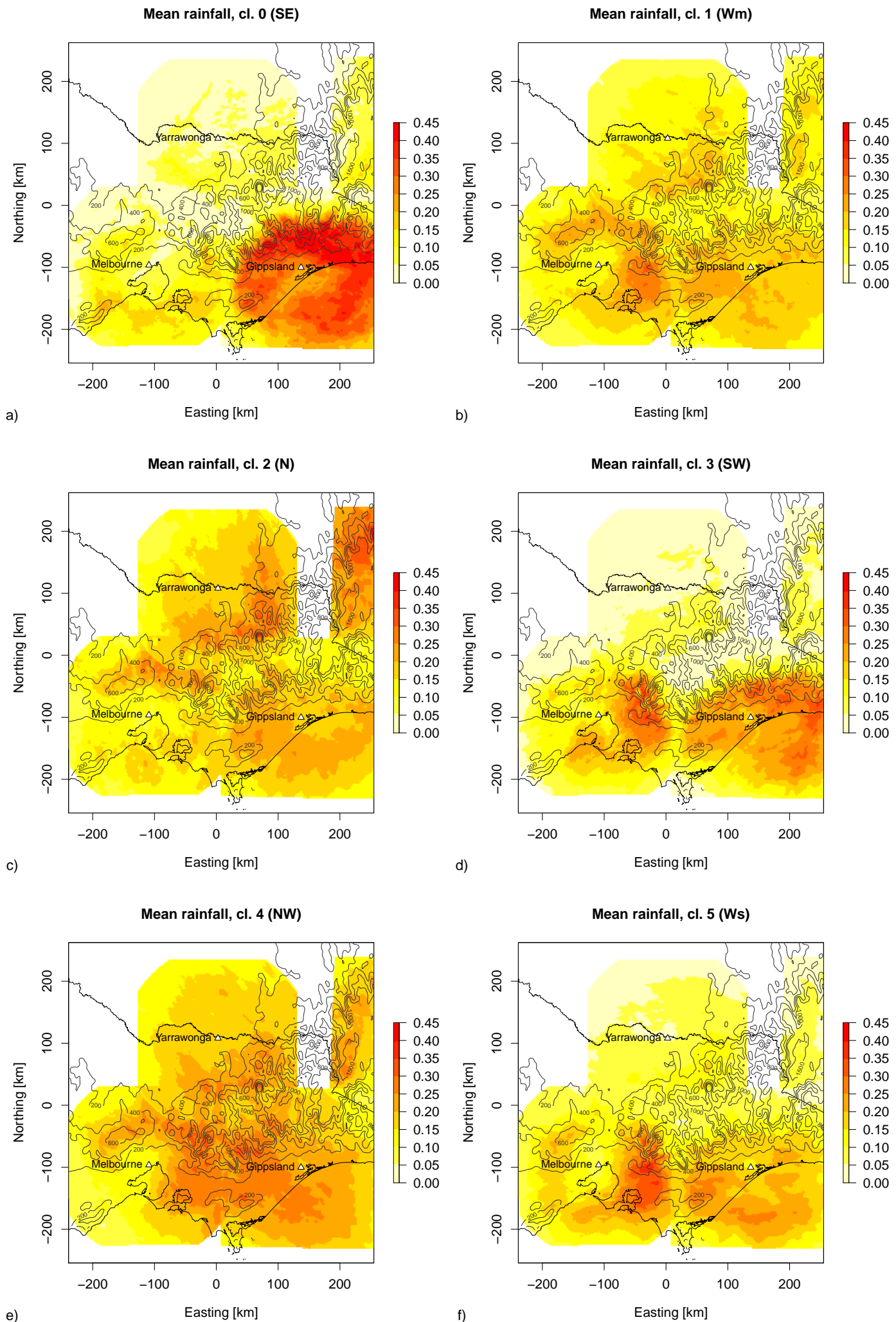

Figure 6. Conditional mean $10 \mathrm{~min}$ rainfall accumulations for flow regimes (a) SE, (b) Wm, (c) N, (d) SW, (e) NW and (f) Ws. 
by flow cluster. Despite some variability arising from periodic features of the Fourier transform, it is possible to notice that lifetimes are higher on the upwind side and lower on the downwind side of terrain features. An illustrative example can be observed under southwesterly conditions (Fig. 7d). The lifetime of precipitation upstream of the Dandenong ranges is about $20-40 \mathrm{~min}$; it increases to $50-70 \mathrm{~min}$ on the upwind side and falls again to 20-30 min when moving into the Alps. Similar patterns can be observed under the flow regime Ws (Fig. 7f). On the other hand, under NW flows short lifetimes are located on the leeward side of the Macedon ranges (Fig. 7e). Note that with reversed flow conditions (SE, Fig. 7a), this region exhibits lifetimes of $80-100 \mathrm{~min}$ and the shortest ones are located on top of the Macedon ranges with values oscillating between 40 and $80 \mathrm{~min}$. The region located South and Southeast of the Yarrawonga radar is also interesting to analyze in particular for the clusters $\mathrm{N}$ and NW. In fact, the location of the longest lifetimes upstream of the Alps is different depending on flow direction (Fig. 7c and e). The plains surrounding the Yarrawonga radar also show very long lifetimes under flow conditions SE, Wm, and SW. However, this effect could be an artefact of the low rainfall accumulations over these regions (see Fig. 6a, b, and d).

These findings corroborate the results of Harris et al. (1996), who demonstrated that the precipitation intermittency is higher upstream compared with the top of the mountain ridge, with intermediate values on the upwind flank. From Fig. 7 it seems that the decreased intermittency of rainfall upwind of orographic features has a positive impact on its predictability by Lagrangian persistence. It is worth mentioning that leeside precipitation enhancement is also possible due to leeside flow convergence, flow perturbations by mountain gravity waves, or the presence of cold air pools that force the unstable air to rise. Such processes are not very frequent and would require stratifying the statistics using more complex criteria based on moist air stability indices among others.

The relationship between the precipitation lifetime and orography is less pronounced than that of nowcast biases presented in Foresti and Seed (2014). This is mostly due to the increased difficulty in computing higher order statistics, which require many more samples than a simple linear or multiplicative bias. Also, the cascade decomposition framework still needs some improvements to reduce the edge effects and to better interpret the intricate statistical dependencies between consecutive cascade levels (see Seed et al., 2013).

\section{Conclusions}

The geographical distribution of the scale-dependent predictability of precipitation by Lagrangian extrapolation of radar images was analyzed under different flow regimes in connection with the presence of orographic features. Data from the Victorian radar composite, Australia, a $500 \times 500 \mathrm{~km}^{2}$ domain covering the period from February 2011 to October 2012, were used for the analyses. The scale dependence of the predictability of precipitation was considered by decomposing the radar rainfall field into a multiplicative cascade using an FFT (Bowler et al., 2006). The lifetime of precipitation features was found to be a power law function of the scale of the features and to depend on flow direction, which confirms the presence of dynamic scaling (Venugopal et al., 1999; Mandapaka et al., 2009). The precipitation lifetime was found to be up to a factor of 2 higher on the upwind compared with the downwind slopes of orographic features and to be strongly flow-dependent. The degree of spatial scaling of the rainfall field was also shown to be spatially inhomogeneous. These spatial heterogeneities due to orographic forcing can be exploited to locally adapt the space-time stochastic simulation of precipitation, which is needed for very short-term forecasting (e.g., Seed et al., 2013), generating radar ensembles (Germann et al., 2009), design storm studies (e.g., Paschalis et al., 2013), and precipitation downscaling (e.g., Pathirana and Herath, 2002).

The study raised several methodological questions, in particular because the quality of radar data is much more homogeneous over time than over space. This has to be accounted for when interpreting the maps of the predictability of precipitation. Some patterns could be simply due to the geographical biases that affect the radar measurements, for example due to beam blockage, signal attenuation, or increasing sampling volume with range. Nevertheless, in the regions close to the radar, it was possible to detect a clear signal in the distribution of the precipitation lifetime, which was attributed to orographic forcing.

The predictability estimates presented in this paper are affected by other sources of uncertainty. The first is related to the assumption of the temporal stationarity of the diagnosed velocity field, which leads to over-optimistic estimates of the precipitation lifetimes, especially at the large scales. The second arises from the uncertainty in the estimation of the velocity field with optical flow. In fact, precipitation fields often show differential motion at different spatial scales. An illustrative example occurs when stationary orographic rainfall contains fast moving cellular convection (e.g., Foresti et al., 2013). Better estimates of the Lagrangian predictability would require the optical flow to be estimated on each spatial scale separately.

Finally, it is not yet clear whether the spatial variability of precipitation lifetime is more significant than its temporal variability, and how to account for both aspects in the real-time nowcasting of precipitation using stochastic simulation approaches such as STEPS. The natural solution would be to allow the predictability to vary through time in a first stage and to gradually add some spatial heterogeneity when more and more radar data are collected. This goal could be achieved by exploiting the online computation of statistics, 


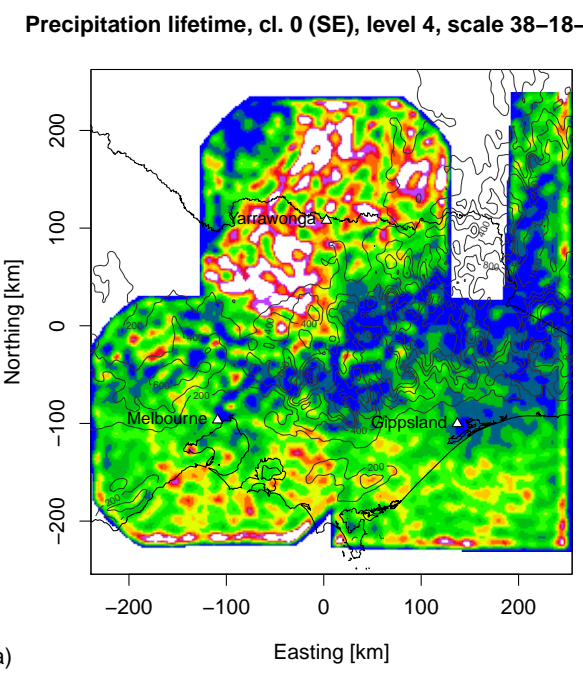

Precipitation lifetime, cl. 2 (N), level 4, scale 38-18-8 km

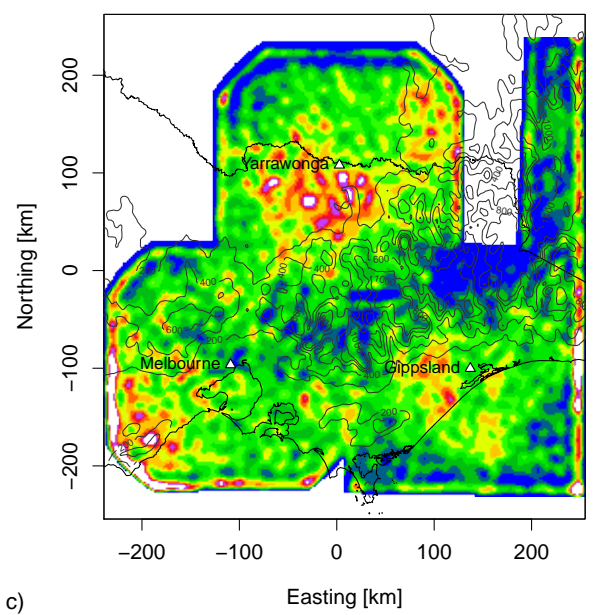

Precipitation lifetime, cl. 4 (NW), level 4, scale 38-18-8 km

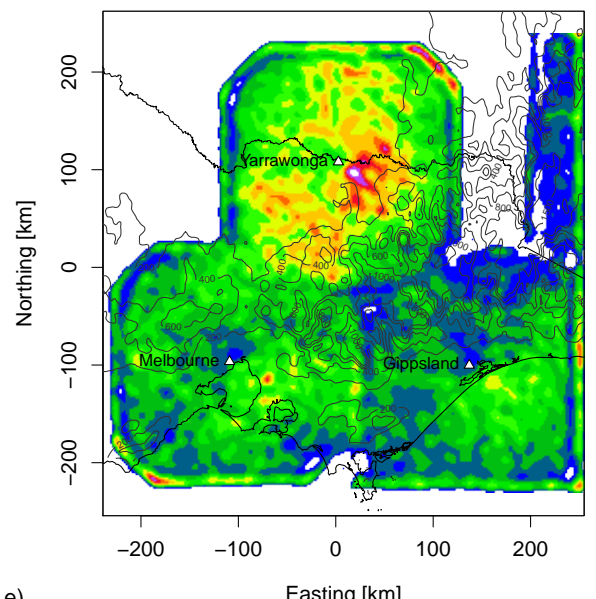

Precipitation lifetime, cl. $1(\mathrm{Wm})$, level 4, scale 38-18-8 km

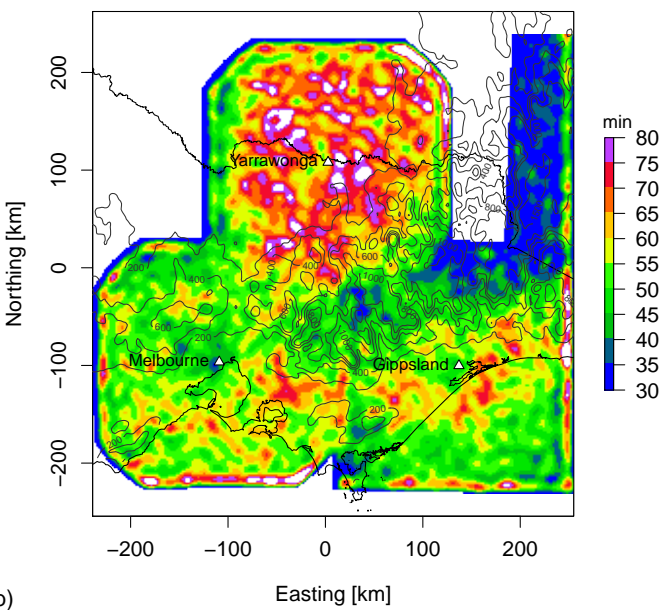

Precipitation lifetime, cl. 3 (SW), level 4, scale 38-18-8 km

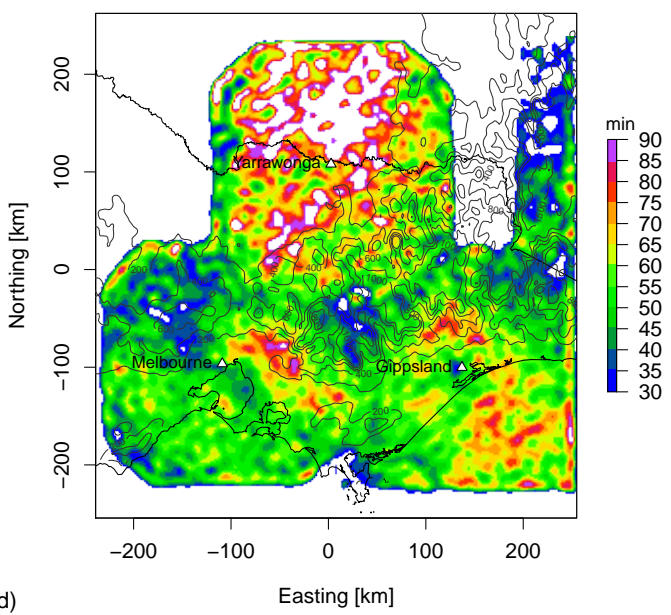

Precipitation lifetime, cl. 5 (Ws), level 4, scale 38-18-8 km

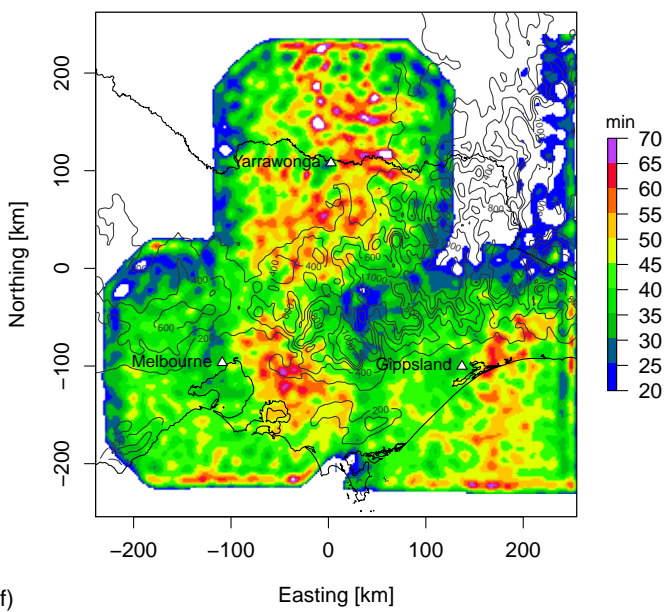

Figure 7. Spatial distribution of the precipitation lifetimes at cascade level 4 (38-18-8 km) stratified by flow regime. (a) SE, (b) Wm, (c) N, (d) SW, (e) NW, (f) Ws. 
which would enable the nowcasting system to learn about the spatial distribution of predictability as more and more radar data are collected and analyzed.

Acknowledgements. This research was funded by the Swiss National Science Foundation (SNSF) project "Data mining for precipitation nowcasting" (PBLAP2-127713/1). We also would like to acknowledge the Belgian Science Policy Office (BELSPO) project PLURISK: "Forecasting and management of rainfall-induced risks in the urban environment" (SD/RI/01A), which allowed this study to be finalized. Urs Germann is specially thanked for the discussion on the predictability of precipitation. We acknowledge Mark Curtis and Kevin Cheong for the technical support received, and Maarten Reyniers and Laurent Delobbe for reviewing the manuscript.

Edited by: H. Leijnse

\section{References}

Badas, M. G., Deidda, R., and Piga, E.: Modulation of homogeneous space-time rainfall cascades to account for orographic influences, Nat. Hazards Earth Syst. Sci., 6, 427-437, doi:10.5194/nhess-6-427-2006, 2006.

Berenguer, M., and Sempere-Torres, D.: Radar-based rainfall nowcasting at European scale: long-term evaluation and performance assessment, Proc. of the 36th AMS Conf. on Radar Meteorology, Breckenridge, Colorado, USA, 2013.

Berenguer, M., Sempere-Torres, D., and Pegram, G. G. S.: SBMcast - An ensemble nowcasting technique to assess the uncertainty in rainfall forecasts by Lagrangian extrapolation, J. Hydrol., 404, 226-240, 2011.

Bousquet, O., Lin, C. A., and Zawadzki, I.: Analysis of scale dependence of quantitative precipitation forecast verification: a casestudy over the Mackenzie river basin, Q. J. Roy. Meteorol. Soc., 132, 2107-2125, 2006.

Bowler, N. E. H., Pierce, C. E., and Seed, A. W.: Development of a precipitation nowcasting algorithm based upon optical flow techniques, J. Hydrol., 288, 74-91, 2004.

Bowler, N. E. H., Pierce, C. E., and Seed, A. W.: STEPS: A probabilistic precipitation forecasting scheme which merges an extrapolation nowcast with downscaled NWP, Q. J. Roy. Meteorol. Soc., 132, 2127-2155, 2006.

Casati, B., Ross, G., and Stephenson, D. B.: A new intensity-scale approach for the verification of spatial precipitation forecasts, Meteorol. Appl., 11, 141-154, 2004.

Chumchean, S., Sharma, A., and Seed, A.: An integrated approach to error correction for real-time radar-rainfall estimation, J. Atmos. Ocean. Tech., 23, 67-79, 2006a.

Chumchean, S., Seed, A., and Sharma, A.: Correcting of real-time radar rainfall bias using a Kalman filtering approach, J. Hydrol., 317, 123-137, 2006b.

Chumchean, S., Seed, A., and Sharma, A.: An operational approach for classifying storms in real-time radar rainfall estimation, J. Hydrol., 363, 1-17, 2008.
Foresti, L. and Seed, A.: On the spatial distribution of rainfall nowcasting errors due to orographic forcing. Meteorol. Appl., doi:10.1002/met.1440, in press, 2014.

Foresti, L., Kanevski, M., and Pozdnoukhov, A.: Kernel-based mapping of orographic rainfall enhancement in the Swiss Alps as detected by weather radar, IEEE T. Geosci. Remote, 50, 2954-2967, 2012.

Foresti, L., Panziera, L., Mandapaka, P. V., Germann, U., and Seed, A.: Retrieval of analogue radar images for ensemble nowcasting of orographic rainfall, Meteoforol. Appl., doi:10.1002/met.1416, in press, 2013.

Germann, U. and Zawadzki, I.: Scale-dependence of the predictability of precipitation from continental radar images, Part I: Methodology, Mon. Weather Rev., 130, 2859-2873, 2002.

Germann, U., Zawadzki, I., and Turner, B.: Scale-dependence of the predictability of precipitation from continental radar images, Part IV: Limits to Prediction, J. Atmos. Sci., 63, 2092-2108, 2006.

Germann, U., Berenguer, M., Sempere-Torres, D., and Zappa, M.: REAL - Ensemble radar precipitation estimation for hydrology in a mountainous region, Q. J. Roy. Meteorol. Soc., 135, 445-456, 2009.

Gires, A., Tchiguirinskaia, I., Schertzer, D., and Lovejoy, S.: Multifractal and spatio-temporal analysis of the rainfall output of the Meso-NH model and radar data, Hydrolog. Sci. J., 55, 380-396, 2011.

Gires, A., Tchiguirinskaia, I., Schertzer, D., and Lovejoy, S.: Influence of the zero-rainfall on the assessment of the multifractal parameters, Adv. Water Resour., 45, 13-25, 2012.

Goormans, T. and Willems, P.: Using local weather radar data for sewer system modeling: case study in Flanders, Belgium, J. Hydrol. Eng., 18, 269-278, 2013.

Grecu, M. and Krajewski, W. F.: A large-sample investigation of statistical procedures for radar-based short-term quantitative precipitation forecasting, J. Hydrol., 239, 69-84, 2000.

Harris, D., Menabde, M., Seed, A., and Austin, G.: Multifractal characterization of rain fields with a strong orographic influence, J. Geophys. Res., 101, 26405-26414, 1996.

Huuskonen, A., Saltikoff, E., and Holleman, I.: The operational radar network in Europe, B. Am. Meteorol. Soc., 95, 897-907, doi:10.1175/BAMS-D-12-00216.1, 2014.

Jordan, P., Seed, A. W., and Weinnman, P. E.: A stochastic model of radar measurement errors in rainfall accumulations at catchment scale, J. Hydrometeorol., 4, 841-855, 2003.

Knuth, D. E.: The Art of Computer Programming, in: volume 2: Seminumerical Algorithms, 3rd Edn, Addison-Wesley, Boston, 1998.

Lorenz, E. N.: Predictability of flow which possesses many scales of motion, Tellus, 21, 289-307, 1969.

Mandapaka, P. V., Lewandowski, P., Eichinger, W. E., and Krajewski, W. F.: Multiscaling analysis of high resolution spacetime lidar-rainfall, Nonlin. Processes Geophys., 16, 579-586, doi:10.5194/npg-16-579-2009, 2009.

Marsan, D., Schertzer, D., and Lovejoy, S.: Causal space-time multifractal processes: Predictability and forecasting of rain fields, J. Geophys. Res., 101, 26333-26346, 1996.

Menabde, M., Harris, D., Seed, A., Austin, G., and Stow, D.: Multiscaling properties of rainfall and bounded random cascades, Water Resour. Res., 33, 2823-2830, 1997. 
Panziera, L. and Germann, U.: The relation between airflow and orographic precipitation on the southern side of the Alps as revealed by weather radar, Q. J. Roy. Meteorol. Soc., 136, 222-238, 2010.

Paschalis, A., Molnar, P., Fatichi, S., and Burlando, P.: A stochastic model for high-resolution space-time precipitation simulation, Water Resour. Res., 49, 8400-8417, doi:10.1002/2013WR014437, 2013.

Pathirana, A. and Herath, S.: Multifractal modelling and simulation of rain fields exhibiting spatial heterogeneity, Hydrol. Earth Syst. Sci., 6, 695-708, doi:10.5194/hess-6-695-2002, 2002.

Pegram, G. G. S. and Clothier, A. N.: High resolution space-time modelling of rainfall: the "String of Beads" model, J. Hydrol., 241, 26-41, 2001a.

Pegram, G. G. S. and Clothier, A. N.: Downscaling rainfields in space and time, using the String of Beads model in time series mode, Hydrol. Earth Syst. Sci., 5, 175-186, doi:10.5194/hess-5175-2001, 2001b.

Press, W. H., Teukolsky, S. A., Vetterling, W. T., and Flannery, B. P.: Numerical Recipes: The Art of Scientific Computing, 3rd Edn., Cambridge University Press, 2007.

Radhakrishna, B., Zawadzki, I., and Fabry, F.: Predictability of precipitation from continental radar images, Part V: growth and decay, J. Atmos. Sci., 69, 3336-3349, doi:10.1175/JAS-D-12029.1, 2012.

Rennie, S. J.: Doppler weather radar in Australia, CAWCR technical report, no. 055, Centre for Australian Weather and Climate Research, Melbourne, Australia, 2012.

Ruzanski, E. and Chandrasekar, V.: An investigation of the shortterm predictability of precipitation using high-resolution composite radar observations, J. Appl. Meteorol., 51, 912-925, 2012.

Schertzer, D. and Lovejoy, S.: Physical modelling and analysis of rain and clouds by anisotropic scaling multiplicative processes, J. Geophys. Res., 92, 9696-9714, 1987.
Seed, A.: A dynamic and spatial scaling approach to advection forecasting, J. Appl. Meteorol., 42, 381-388, 2003.

Seed, A. and Pegram, G. G. S.: Using Kriging to infill gaps in radar data due to ground clutter in real-time. Proceedings of the 5th Int. Symp. on Hydrological Applications of Weather Radar, Kyoto, Japan, 73-78, 2001.

Seed, A., Duthie, E., and Chumchean, S.: Rainfields: the Australian Bureau of Meteorology system for quantitative precipitation estimation, Proc. of the 33rd Conf. on Radar Meteorology, Cairns, Australia, 2007.

Seed, A. W., Pierce, C. E., and Norman, K.: Formulation and evaluation of a scale decomposition-based stochastic precipitation nowcast scheme, Water Resour. Res., 49, 6624-6641, 2013.

Sinclair, S. and Pegram, G. G. S.: Empirical Mode Decomposition in 2-D space and time: a tool for space-time rainfall analysis and nowcasting, Hydrol. Earth Syst. Sci., 9, 127-137, doi:10.5194/hess-9-127-2005, 2005.

Surcel, M., Zawadzki, I., and Yau, M. K.: On the filtering properties of ensemble averaging for storm-scale precipitation forecasts, Mon. Weather Rev., 142, 1093-1105, 2014.

Turner, B. J., Zawadzki, I., and Germann, U.: Predictability of precipitation from continental radar images, Part III: operational nowcasting implementation (MAPLE), J. Appl. Meteorol., 43, 231-248, 2004.

Venugopal, V., Foufoula-Georgiou, E., and Sapozhnikov, V.: Evidence of dynamic scaling in space-time rainfall, J. Geophys. Res., 104, 31599-31610, 1999.

Zawadzki, I. I.: Statistical properties of precipitation patterns, J. Appl. Meteorol., 12, 459-472, 1973.

Zawadzki, I., Morneau, J., and Laprise, R.: Predictability of precipitation patterns - an operational approach, J. Appl. Meteorol., 33, 1562-1571, 1994. 